\title{
Topographic Lectin Mapping of the Epithelial Cell Surface in Normal Intestine and Celiac Disease
}

\author{
Hugh James Freeman* \\ Department of Medicine (Gastroenterology), University of British Columbia, Vancouver, BC, Canada \\ *Corresponding author: hugfree@shaw.ca \\ Received September 16, 2019; Revised October 17, 2019; Accepted December 04, 2019
}

\begin{abstract}
The etiology and pathogenesis of celiac disease remains obscure but remains the focus of intense research investigation. It is generally believed to be an immune-mediated small intestinal mucosal disorder that can cause diarrhea, impaired nutrient assimilation and weight loss. A key component in this process occurs at the intestinal epithelial cell surface that is closely associated with the luminal intestinal microbiome. Here, epithelial membrane glycoproteins and glycolipids are present along with adsorbed molecules that permit interaction with the intestinal microbiome. In recent years, use of specific sugar residue seeking proteins, lectins, that can be found in the diet have been employed topographically to map the small intestinal cell surface and goblet cell secretory mucins to further elucidate the structure and function of this tissue. Evidence has accumulated to indicate that this microenvironment may be critically important in further understanding the etiology and pathogenesis of celiac disease and other sprue-like intestinal disorders.
\end{abstract}

Keywords: lectins, celiac disease, lectin hypothesis, glycoproteins, microbiome

Cite This Article: Hugh James Freeman, "Topographic Lectin Mapping of the Epithelial Cell Surface in Normal Intestine and Celiac Disease.” International Journal of Celiac Disease, vol. 7, no. 3 (2019): 69-73. doi: 10.12691/ijcd-7-3-3.

\section{Overview of Lectins}

Lectins are naturally occurring proteins or glycoproteins that are ubiquitous and largely derived from plants. They are often found in foods, including common items like peas, beans, potatoes, soybeans, tomatoes and wheat germ. Lectins have been shown to have multiple effects. At least some lectins are known to be potent biological agents that bind to specific sugar residues along the length of the intestinal tract, cause agglutination of fetal and neoplastic cells, inhibit in vivo and in vitro tumor growth and stimulate lymphocyte proliferation [1]. The precise role of lectins in nature is still not completely clear, but these proteins serve in biological recognition and mediate attachment and binding of bacteria and viruses to intended targets. Some lectins may serve a beneficial role while others, like ricin, may serve as potent toxins, even utilized by terrorists. In the past, the sugar specificity of lectins has been useful in the analysis of blood types as well as transfer of traits in genetic engineering of crops to promote resistance to pests and herbicides.

\section{Transit in the Intestinal Tract}

Their presence in food has resulted in studies of the disposition of lectins within the human intestine. During mastication in the oral cavity, and later, during digestion, lectins may be released from food and remain intact during passage as far as the distal small intestine [2]. Information on their role in the digestive process, however, is largely unknown and their effect on the assimilation of both micro- and macro-nutrients is limited.

\section{Lectin Effects on Nutrient and Drug Absorption}

The rate of absorption of carbohydrate from the intestinal lumen may be altered by the presence of different foods, including vegetable proteins, fats, fibre and so-called "anti-nutrients", specifically, phytates and lectins [3]. A recent review [4] outlined the potential anti-nutritive and even toxic effects of plant lectins, largely related to their ability to bind to specific carbohydrates on membrane glycoproteins and glycolipids integral to the luminal cell surface border of intestinal epithelial cells as well as their ability to survive digestive processes in the gastrointestinal tract. Turnover of epithelial cells may be affected by luminal lectin activities causing impaired nutrient digestion and absorption, changes in luminal bacterial flora and the intestinal immune state. At high intakes, lectins may have significant effects on normal growth and the health of different animal species. Interestingly, recent reports note that specific lectins may mediate muco-adhesion, cyto-adhesion and cyto-invasion of some orally consumed drugs and other therapeutic agents. Future investigative studies may potentially lead to improved drug absorption 
strategies and bioavailability of several drugs, peptides and proteins that are normally poorly absorbed [5].

\subsection{Sugar Specificity of Lectins in the Small Intestine}

These proteins exhibit in vivo and in vitro specificity for specific sugar residues. Table 1 shows some examples along with the specific binding inhibitor molecule(s) [6,7]. In the intestinal tract, a number of studies have shown that lectins, if labeled with flourescein or peroxidase, may be used to histochemically "map" the topography of mammalian, including human, intestine in different conditions or diseases, including celiac disease [7]. In this setting, lectins are thought to largely bind to glycoprotein and glycolipid molecules integral or attached to the epithelial cell membranes as well as to mucus glycoproteins found in goblet cells (Figure 1). Not shown in this schematic figure, but also critically important, are adsorbed carbohydrate containing molecules that may have been sourced from elsewhere in the intestinal tract or include components from ingested food and other luminally-digested proteins, including intra-luminal bacteria, namely the intestinal luminal microbiome [8]. Prior studies have demonstrated sharp contrasts in the patterns of binding in different intestinal regions, including the normal small bowel and colon [6,7]. Some of these binding patterns using fluorescein-labeled lectins are shown in Figure 2 and Figure 3 for the small intestine, specifically duodenum, and Figure 4 for a small intestinal vascular structure. As shown, specific patterns of labeling occur with different lectins, possibly reflecting the presence of different intestinal cell molecular structures and/or differences in the microbiome. Figure 2a shows the villus structure of mammalian small intestine with bright goblet cell and epithelial cell surface labeling after application of wheat germ agglutinin. A similar pattern, shown in Figure 2b, is evident with the same lectin for the crypt region. Interestingly, in the crypts, Paneth cells can be easily distinguished from other epithelial cell types in the crypt bases. In contrast, Figure 3a shows a different lectin, ricin, binding in the villus region with predominately cell surface staining and an absence of goblet cell mucus staining. Figure $3 \mathrm{~b}$ has a similar binding pattern for ricin with distinct cell surface staining while goblet cell mucus staining is not evident. Of interest, Figure 4 shows the staining pattern for a single vascular structure in the small intestinal mesentery suggesting that future investigative studies may not necessarily be limited to the intestinal luminal surface. Most distinctive is the elastic layer in the muscle wall, and, both intravascular red cells and white cells are also present.

Table 1. Carbohydrate specificity for some lectins

\begin{tabular}{llll}
\hline Lectin & Plant Origin & Major Sugar Specificities* & Binding Inhibitor*\# \\
\hline Wheat germ (WGA) & Triticum vulgare & (GlcNAc)n; NeuNAc & GlcNAc \\
Castor bean (RCA1) & Ricinus communis & Gal & Gal \\
Gorse (UEA1) & Ulex europeus & L-Fuc & L-Fuc \\
Horsegram (DBA) & Dolichos biflorus & GalNAc & GalNAc \\
Jackbean (ConA) & Canavalia ensiformis & Man; Glc & MM \\
Peanut (PNA) & Arachis hypogea & Gal-GaNAc>Gal & Gal \\
\hline
\end{tabular}

*L-Fuc, alpha-L-fucose; MM, alpha-methyl-mannoside; GalNAc, N-acetyl-galactosamine; Glc, glucose; GlcNAc, N-acetylglucosamine; Man, mannose; NeuNAc,N-acetyl-neuraminic acid (sialic acid). All sugars are beta-D configuration unless otherwise stated. \#To inhibit lectin binding, sugars used at $0.2 \mathrm{M}$ except for PNA binding which requires 0.3M [6,7].

Table 2. Semi-quantitative Lectin Luminal Surface Labeling

\begin{tabular}{|c|c|c|c|c|c|}
\hline \multirow[t]{2}{*}{ Lectin } & \multicolumn{2}{|c|}{ Controls } & \multirow[b]{2}{*}{$\underline{\text { Villus }}$} & \multicolumn{2}{|c|}{$\square$ Celiacs } \\
\hline & Crypt Base & Crypt Apex & & Crypt Base & Crypt Apex \\
\hline WGA & $0.2+0.1$ & $1.8+0.6$ & $2.7+0.4^{*}$ & $1.8+0.2$ & $3.8+0.1^{*}$ \\
\hline RCA1 & $2.0+0.4$ & $3.0+0.3$ & $3.0+0.3^{*}$ & $1.6+0.2$ & $2.3+0.2 *$ \\
\hline UEA1 & $1.3+0.4$ & $1.5+0.5$ & $2.0+0.5^{*}$ & $3.5+0.5$ & $3.6+0.3$ \\
\hline DBA & $0.2+0.2$ & $0.5+0.3$ & $0.8+0.4$ & $0.4+0.2$ & $0.9+0.3$ \\
\hline ConA & $0.2+0.2$ & $1.6+0.4$ & $2.4+0.3^{*}$ & $0.1+0.1$ & $1.6+0.2^{*}$ \\
\hline
\end{tabular}

Range of observations of fluorescence intensity from 0 to 4 with Mean+SEM for control (n=6) and celiac (n=6) biopsies as per Reference 6. For PNA, consistent labeling not present. $* \mathrm{P}<0.05$ compared to crypt base.

Table modified from Reference [7].

Table 3. Semi-quantitative Goblet Cell Labeling

\begin{tabular}{|c|c|c|c|c|c|}
\hline \multirow[t]{2}{*}{ Lectin } & \multicolumn{2}{|c|}{ Controls } & \multirow[b]{2}{*}{ Villus } & \multicolumn{2}{|c|}{ Celiacs } \\
\hline & Crypt Base & Crypt Apex & & Crypt Base & Crypt Apex \\
\hline WGA & $0.2+0.1$ & $1.7+0.4$ & $2.7+0.3^{*}$ & $1.1+0.2$ & $3.6+0.2 *$ \\
\hline RCA1 & $0.5+0.3$ & $2.7+0.3^{*}$ & $2.7+0.3^{*}$ & $0.2+0.1$ & $3.3+0.1^{*}$ \\
\hline UEA1 & $0.3+0.2$ & $1.5+0.4^{*}$ & $1.5+0.4^{*}$ & $1.1+0.3$ & $2.7+0.3$ \\
\hline DBA & $0.2+0.2$ & $1.5+0.5$ & $1.5+0.5$ & $1.1+0.2$ & $0.8+0.2$ \\
\hline ConA & $0.2+0.2$ & $0.4+0.2$ & $0.4+0.2$ & $0.1+0.1$ & $1.3+0.4^{*}$ \\
\hline
\end{tabular}

Range of observations of fluorescence intensity from 0 to 4 with Mean+SEM for control (n=6) and celiac (n=6) biopsies as per Reference 6. For PNA, consistent labeling not present. ${ }^{*} \mathrm{P}<0.05$ compared to crypt base.

Table modified from Reference [7]. 


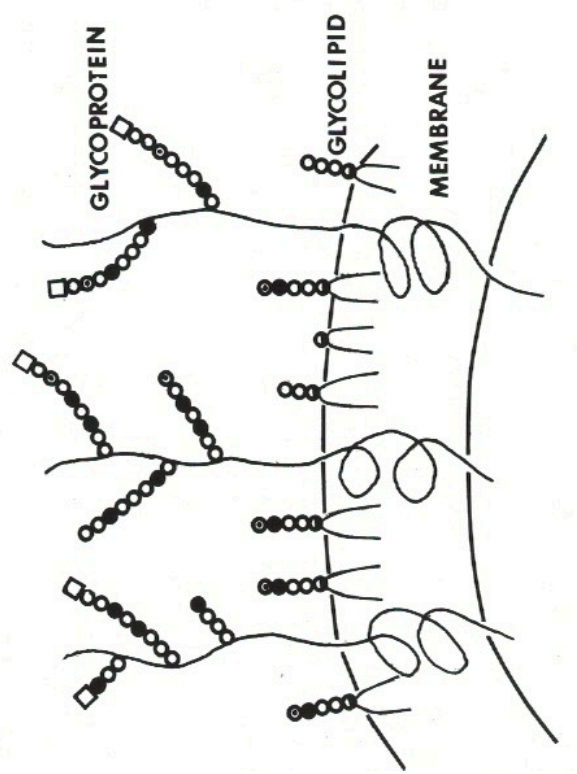

Figure 1. Schematic representation of intestinal epithelial cell surface showing carbohydrate containing molecules including glycolipids and glycoproteins, some passing through the bilayer membrane as transmembrane proteins. The carbohydrate or sugar residues are oriented towards the lumen of the intestine. Not shown are similar structures that adsorb or adhere to the epithelial cell surface. These may be intrinsic to the underlying intestinal cell or extrinsic from the diet, luminal bacteria or other sloughed proteins

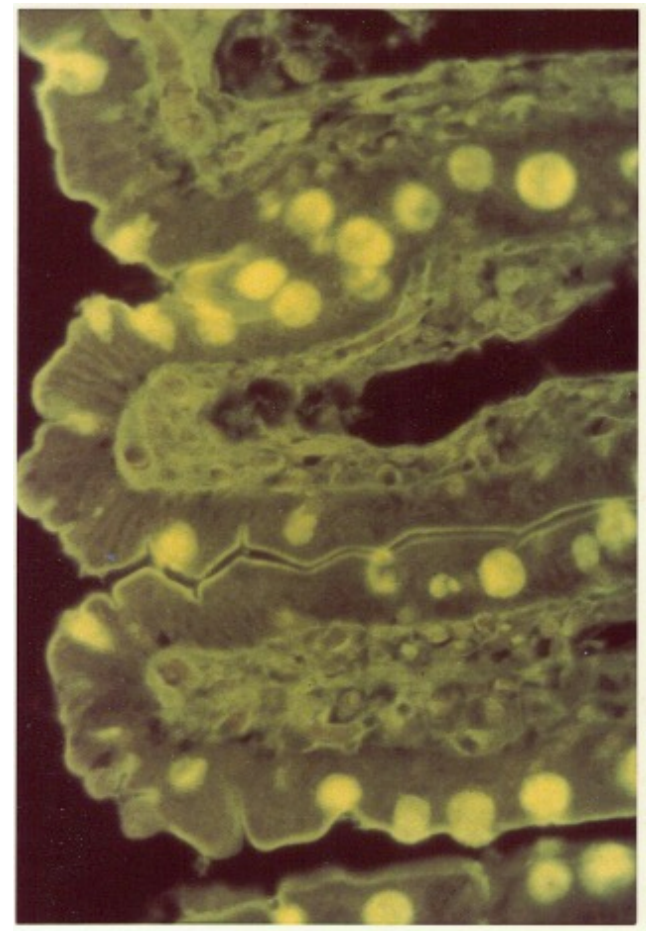

Figure 2a. Normal small intestinal villus showing cell surface and goblet cell carbohydrate-containing material labeled with fluoresceinconjugated wheat germ agglutinin (WGA)

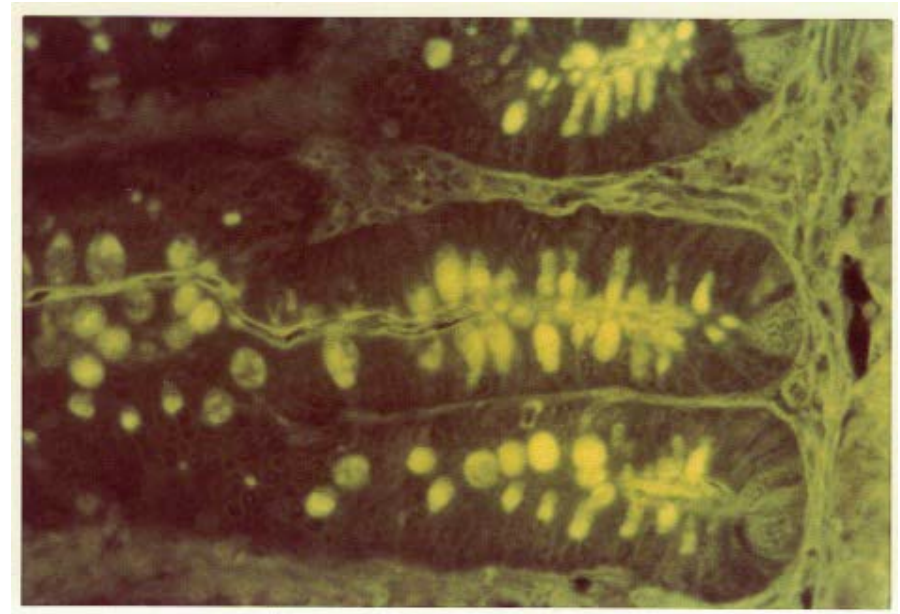

Figure 2b. Normal small intestinal crypt region also labeled with fluorescein-conjugated WGA. Paneth cells are also observed at the base of the crypts

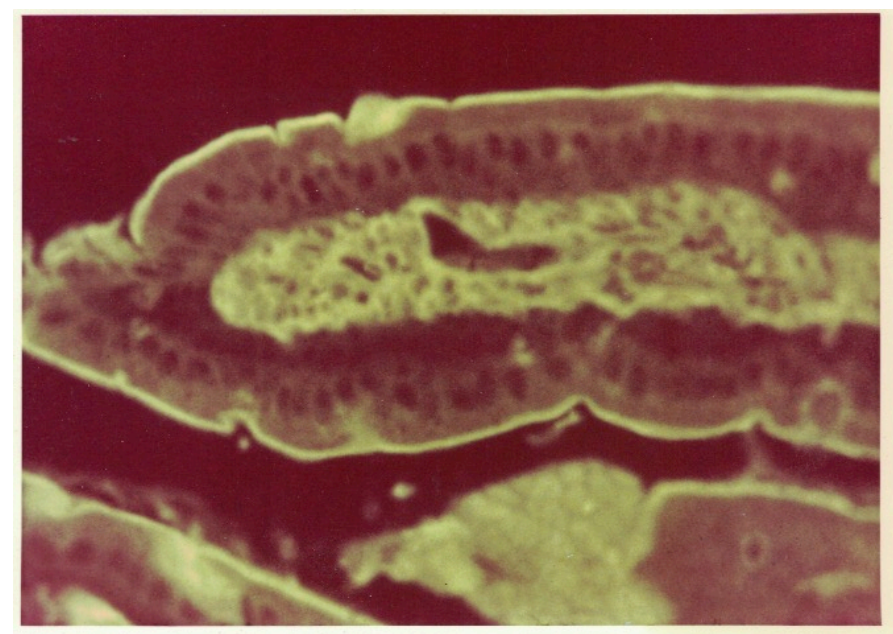

Figure 3a. Normal small intestinal villus labeled with fluorescein-conjugated ricin communis (RCA1). The pattern of labeling is distinct from WGA 


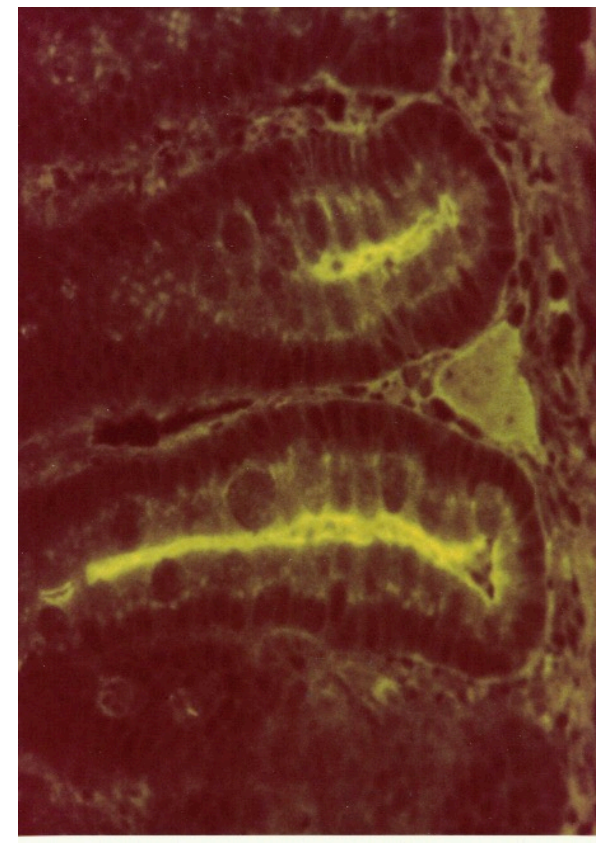

Figure 3b. Normal small intestinal crypt region also labeled with RCA1. Note distinct pattern of labeling with binding to cell surface but not the goblet cells

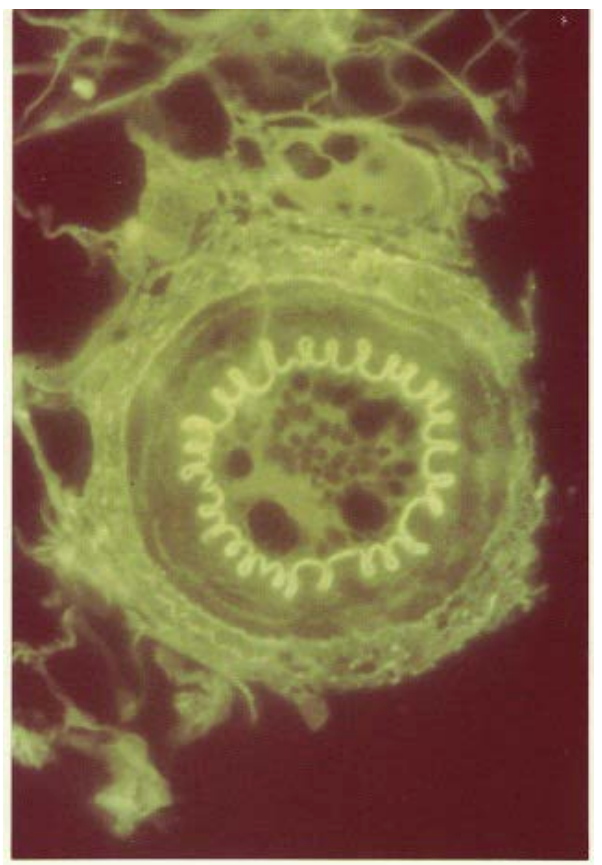

Figure 4. Cross-section of mesenteric blood vessel showing lectin labelled vessel along with intravascular hematologic elements

\subsection{Celiac Disease and the Lectin Hypothesis}

Similar lectin methods have been applied in celiac disease. Celiac disease is an immune-mediated small intestinal disorder that improves with restriction of dietary gluten. The disorder is characterized by impaired nutrient absorption, malnutrition and weight loss. The precise cause of celiac disease is unknown although it appears that predisposition to the disease is genetically-based. Possible mechanisms leading to the characteristic histopathological lesion still require elucidation.

Interestingly, wheat germ agglutinin, a lectin with sugar specificity for N-acetyl-D-glucosamine, also can be detected in commercial preparations of gluten powder and antibodies to wheat germ agglutinin have been reported in celiac disease [9]. In the past, it was hypothesized that gluten may cause innate cell toxicity by acting as a lectin binding agent to defective brush border membrane glycoproteins [10]. Moreover, lectin activity identified as wheat germ was reported in gluten [11]. Similarly, serum antibodies to wheat germ agglutinin and gluten were reported in dermatitis herpetiformis, a skin disorder closely linked to celiac disease [12]. A similar lectin hypothesis was also suggested for soy protein intolerance that was thought to cause a clinically and histologically indistinguishable picture to untreated celiac disease [13]. Although interesting, these observations have not been considered sufficient to provide definitive evidence of a relationship, if any, that the lectin hypothesis may contribute to improved understanding of the cause of celiac disease. However, this and other novel methods utilizing lectins may be helpful in pursuing some aspects of the pathogenesis of the disorder, especially since lectins do have the ability to mark specific individual sugar residues in or attached to the surface structures of the epithelial cells in different stages of differentiation. Using specific lectins, marked differences in the intensity and patterns of binding were noted in both control and celiac disease biopsies [7], particularly along the crypt-to-villus column, reflecting the increasing epithelial cell differentiation. These changes were observed in control adult normal biopsies from the crypt epithelial cell surface compared to the villus epithelial cell surface. This appears to be consistent with the concept that epithelial cells migrate from the crypt to villus region as enterocyte differentiation proceeds and increasing epithelial cell maturation occurs. During this migration, sugar residues appear to be sequentially added to partially completed oligosaccharide structures to form fully completed oligosaccharide side chains.

Most intriguing, there were also changes along the crypt epithelial cell surface in celiac biopsies from adult small intestine. Although it is believed that in celiac disease, crypt cells are less well differentiated epithelial cells, the crypt epithelial cell surface also appears to undergo a differentiation process in both control and celiac biopsies. While this differentiation process may differ quantitatively and/or qualitatively from normal epithelial cells, crypt cells in celiac disease also appear to be able to sequentially add sugar residues in developing cells as these migrate from the base of the crypts to the small intestinal lumen. This altered pattern of labeling with lectins in celiac disease may not be specific for celiac disease per se but might result from any form of injury or toxicity that alters the structure of the small intestine [14]. Similar labeling changes have also been reported by others [15]. Perhaps, the specificity of these changes could be explored in future in other "sprue-like" disorders that may cause a similar histopathologic appearance (eg., olmesartan enteropathy) [16,17]. Biopsies from children with celiac disease have also been compared to controls including post-enteritis syndrome [18,19,20].

Even with this lectin binding data, the pathogenesis of celiac disease remains elusive. Presumably, in geneticallypredisposed persons, gluten (or perhaps, one of its components) initiates a toxic reaction by binding to intestinal surface structures. Older studies hypothesized 
that a deficiency of intestinal peptidase activity caused toxic gluten degradation products to bind to intestinal surface structures followed by immunoregulatory pathomechanisms that might damage the intestinal mucosa. Alternatively, a change induced in the microbiome might result in similar effects [21].

Finally, other cells, including intestinal stem cells [22] and Paneth cells (including their alpha-defensins), if altered by lectin binding activities, may provide a key role in linking the microbiome with celiac disease [23]. Previously, several investigators [24,25,26] hypothesized that gluten acts as a lectin, binding to specific oligosaccharide structures of the intestinal epithelial cell surface and causing cell toxicity, but this hypothesis has been considered to be controversial $[20,27]$. Some recent evidence has accumulated to show that gliadin and gluten peptides may behave like lectins with the ability to bind to high mannose-type glycoproteins in human serum as well as immature crypt cells in rat intestine [24,25]. Gliadin peptides from wheat may reversibly agglutinate poorly differentiated human K562(S) cells. This binding and agglutinating activity was apparently inhibited by mannan, a mannose polysaccharide and N-acetyl-glucosamine [28]. Also, mannan and $\mathrm{N}$-acetyl-glucosamine oligomers were reported to exhibit protective effects to gliadin in vitro using cultured small intestinal biopsies from children with active celiac disease [29]. Further studies are still needed to determine if a unique and specific lectin binding event in celiacs occurs that might provoke a toxic mucosal reaction. More recently, the role of mannose-binding lectin has been explored in celiac disease with genotype analysis. Evidence was accumulated for a higher frequency of mutant $0 / 0$ in Italian patients with celiac disease compared to pan-ethnic healthy controls [28]. Subsequently, the same investigative group has provided further evidence of a correlation with mannose binding lectin and celiac disease, and possibly, other autoimmune diseases. As recently noted, mannose-binding lectin status remains a research tool [29], although replacement therapy and use in disease prediction may appear in the near future.

\section{References}

[1] Lis H, Sharon N. The biochemistry of plant lectins (phytohemagglutinins). Ann Rev Biochem 1973; 42: 541-574.

[2] Brady PG, Vannier AM, Banwell JG. Identification of the dietary lectin, wheat germ agglutinin, in human intestinal contents. Gastroenterology 1978; 75: 236-239.

[3] Jenkins DJ, Josse RG, Jenkins AL, Wolever TM, Vuksan V. Implications of altering the rate of carbohydrate absorption from the gastrointestinal tract. Clin Invest Med 1995; 18: 296-302 [PMID 8549016].

[4] Vasconceios IM, Oliveira JT. Antinutrtional properties of plant lectins. Toxicon 2004; 44: 385-403.

[5] Gabor F, Bogner E, Weissenboeck A, Wirth M. The lectin-cell interaction and its implications to intestinal-lectin mediated drug delivery. Adv Drug Deliv Rev 2004; 56: 459-480.

[6] Freeman HJ, Lotan R, Kim YS. Application of lectins for detection of goblet cell glycoconjugate differences in proximal and distal colon of the rat. Lab Invest 1980; 42: 405-412.
[7] Freeman HJ. Topography of lectin binding sites in celiac sprue. Can J Gastroenterol 1992; 6: 271-276.

[8] Lynch SV, Pedersen O. The human intestinal microbiome in health and Disease. N Engl J Med 2016; 375: 2369-2379.

[9] Solid LM, Kolberg J, Scott H, Ek J, Fausa O, Brandtzaeg P. Antibodies to wheat germ agglutinin in celiac disease. Clin Exp Immunol 1986; 63: 95-100.

[10] Weiser MM, Douglas AP. An alternative mechanism for gluten toxicity in celiac disease. Lancet 1976; i: 567-569.

[11] Kollberg J, Sollid L. Lectin activity of gluten identified as wheat germ Agglutinin. Biochem Biophys Res Comm 1985; 130:867.

[12] Solid LM, Scott H, Kolberg J, Brandtzaeg P. Serum antibodies to wheat germ agglutinin and gluten in patients with dermatitis herpetiformis. Arch Dermatol Res 1986; 278: 433-436.

[13] Ament ME, Rubin CE. Soy protein-another cause of the flat intestinal lesion. Gastroenterology 1972; 62: 227-234.

[14] Vecchi M, Tornano G, de Franchis R, Tronconi S, Agape D, Ronchi G. Evidence of altered structural and secretory glycoproteins in the jejunal mucosa of patients with gluten sensitive enteropathy and subtotal villus atrophy. Gut 1989; 30: 804-810.

[15] Barresi G, Tuccari G, Tedeschi A, Magazzu G. Lectin binding sites in duodeno-jejunal mucosas from celiac children. Histochemistry 1988; 88: 105-112.

[16] Freeman HJ. Sprue-like intestinal disease. Int J Celiac Dis 2014; 2:6-10.

[17] Freeman HJ. Olmesartan enteropathy. Int J Celiac Dis 2016; 4: 24-26.

[18] Pittschieler K, Ladinser B, Petell JK. Reactivity of gliadin and lectins with celiac intestinal mucosa. Pediatr Res 1994; 36: 635-641.

[19] Toft-Hansen H, Neilson C, Biagini M, Husby S, Lillevang ST. Lectin staining shows no evidence of involvement of glycocalyx/mucus layer carbohydrate structures in development of celiac disease. Nutrients 2013; 5: 4540-4552.

[20] Cenit MC, Olivares M, Codoner-Franch P, Sanz Y. Intestinal microbiota and celiac disease: cause, consequence or co-evolution? Nutrients 2015; 7: 6900-6923.

[21] Piscaglia AC. Intestinal stem cells and celiac disease. World J Stem Cells 2014; 6: 213-229.

[22] Nakamura K, Sakuragi N, Takakuwa A, Ayabe T. Paneth cell alpha-defensins and enteric microbiota in health and disease. Biosci Microbiota Food Health 2016; 35: 57-67.

[23] Douglas AP. The binding of the glycopeptide component of wheat gluten to intestinal mucosa of normal and celiac human subjects. Clin Chim Acta 1976; 73: 357-361.

[24] Kottgen E, Volk B, Kluge F, Gerok W. Gluten, a lectin with oligo-mannosyl specificity and the causative agent of glutensensitive enteropathy. Biochem Biophys Res Commun 1982; 109: 168-173.

[25] Kottgen E, Kluge F, Volk B, Gerok W. The lectin properties of gluten as basis of the pathomechanism of gluten-sensitive enteropathy. Klin Wochenschr 1983; 61: 111-112.

[26] Colyer J, Farthing MJ, Kumar PJ, Clark ML, Ohannesian AD, Waldron NM. Reappaisal of the "lectin hypothesis in the etiopatho-genesis of celiac disease. Clin Sci (Lond) 1986; 71: 105-110.

[27] Ruhlmann J, Sinha P, Hansen G, Tauber R, Kottgen E. Studies on the etiology of celiac disease: no evidence for lectin-like components in wheat gluten. Biochim Biophys Acta 1993; 1181: 249-256.

[28] Auricchio S, De Rites G, De Vincenzi M. Agglutinating activity of gliadin-derived peptides from bread wheat: implication for celiac disease pathogenesis. Biochem Biophys Res Comm 1982; 109: 168-173.

[29] Auricchio S, De Rites G, De Vicenzi M. Mannan and oligomers of $\mathrm{N}$-acetyl-glucosamine protect intestinal mucosa of celiac patients with active disease from in vitro toxicity of gliadin peptides. Gastroenterol 1990; 99: 973-978. 